\title{
Introduction to the Virtual Issue: Innovative Methods in Online Research
}

\author{
R. Michael Alvarez \\ Division of Humanities and Social Sciences \\ California Institute of Technology \\ Pasadena, CA 91125 \\ email:rma@hss.caltech.edu
}

The pace at which new innovations arise in social science research these days is staggering. It seems that nearly every day there is an impressive new study, based on a clever use of data from new communication technologies, or utilizing impressive computational facilities.

In order to draw attention to this innovative new work, we have put together our eighth $\underline{\text { Political }}$ Analysis virtual issue. This new virtual issue contains six impressive and innovative papers recently published in the journal. Each of these papers uses new data or new computational approaches to address important questions in political science.

The papers that appear in this virtual issue, titled Innovative Methods in Online Research, are all available for free access for a limited time. The papers in this virtual issue are:

- Pablo Barbera, "Birds of the Same Feather Tweet Together: Bayesian Ideal Point Estimation Using Twitter Data" (doi:10.1093/pan/mpu011).

- Andrew M. Guess, "Measure for Measure: An Experimental Test of Online Political Media" (doi:10.1093/pan/mpu010).

- Adam J. Berinsky, Gregory A. Huber, and Gabriel S. Lenz, "Evaluating Online Labor Markets for Experimental Research: Amazon.com's Mechanical Turk" (doi:10.1093/pan/mpr057).

- Zack W. Almquist and Carter T. Butts, "Dynamic Network Logistic Regression: A Logistic Choice Analysis of Inter- and Intra-Group Blog Citation Dynamics in the 2004 US Presidential Election" (doi: 10.1093/pan/mpt016).

- Vito D'Orazio, Steven T. Landis, Glenn Palmer and Philip Schrodt, "Separating the Wheat from the Chaff: Applications of Automated Document Classification Using Support Vector Machines" (doi: 10.1093/pan/mpt030).

- Christopher Lucas, Richard A. Nielsen, Margaret E. Roberts, Brandon M. Stewart, Alex Storer, and Dustin Tingley, "Computer assisted text analysis for comparative politics" (doi: 10.1093/pan/mpu019).

Each of these innovative papers is contributing in both methodological and substantive ways to social science.

Today, vast quantities of information are generated daily by social media -- for example, on blogs, Facebook, and Twitter. This vast quantity of data presents the social science researcher with many new ways to study many important questions; but this vast quantity of data also poses 
considerable methodological problems, a selection of which are considered by four of the papers in this virtual issue. For example, Barbera's paper shows how information gleaned from Twitter messages can be used to estimate ideological ideal points, while Almquist and Butts use information from blogs to model political interaction networks online. Two of the other papers develop techniques for analyzing similar textual information to study social phenomenon, the papers by D'Orazio et al. and Lucas et al.

The other two papers in this virtual issue show how new online technologies can be used by the social scientist for experimentation. Both Berinksy et al., and Guess, explicate the utility of online experiments for social science research. These tools are being used by social scientists for their research, and by practitioners in their work. Online experiments have great potential as tools for social science research, and both of these papers provide a wealth of insight into these new tools.

It's exciting as a journal editor to have an opportunity to have papers as innovative and important as these published in our journal. These papers all demonstrate that social scientists are doing impressive research with the novel data that is being generated in vast quantities every day --and that methodologists are working to develop new ways to analyze all of this new data. The six papers in this virtual issue provide a strong foundation for what is developing into a fascinating new domain of social science research, both substantively and methodologically. 